\title{
Adaptive Fuzzy Consensus Tracking Control for Nonlinear Multiagent Systems with Time-Varying Delays and Constraints
}

\author{
Jie $\operatorname{Lan}^{1,2}$ and Tongyu Xu $\mathbb{D}^{1}$ \\ ${ }^{1}$ College of Information and Electrical Engineering, Shenyang Agricultural University, Shenyang 110000, Liaoning, China \\ ${ }^{2}$ College of Science, Liaoning University of Technology, Jinzhou 121001, Liaoning, China \\ Correspondence should be addressed to Tongyu Xu; xutongyu@syau.edu.cn
}

Received 31 March 2021; Revised 25 April 2021; Accepted 9 June 2021; Published 29 June 2021

Academic Editor: Xiaodi Li

Copyright (c) 2021 Jie Lan and Tongyu Xu. This is an open access article distributed under the Creative Commons Attribution License, which permits unrestricted use, distribution, and reproduction in any medium, provided the original work is properly cited.

\begin{abstract}
This paper proposes an adaptive fuzzy distributed consensus tracking control scheme for a class of uncertain nonlinear dynamic multiagent systems (MASs) with state time-varying delays and state time-varying constraints. The existing controllers with Lyapunov-Krasovskii functions (LKFs) were not suitable to address time-varying delays and time-varying constraints in nonlinear MASs simultaneously. State constraints further increase the difficulty of controller design and stability analysis, especially for nonstrict feedback systems. Fuzzy logic systems (FLSs) tackle the approximation of unknown dynamics functions and parameters. Especially when the distributed consensus tracking error is infinitely close to the origin, although there is no singular value, it would lead to the rapid growth of control rate or uncontrollability. Constructing appropriate piecewise functions can effectively avoid the above occurrence and accelerate convergence. Based on Lyapunov stability theory and algebraic graph theory, the constructed tracking control can ensure states within defined time-varying constraint bounds and eliminate the influence of time delays. All signals in closed-loop systems can be guaranteed semiglobally uniformly ultimately bounded (SUUB). Finally, the validity of the theoretical method is verified by the simulation.
\end{abstract}

\section{Introduction}

Over the past few decades, inspired by flocking behavior in nature, related research on multiagent systems (MASs) had developed rapidly due to potential military and civilian applications. The literature involved many aspects such as satellites, flights, distributed computing, robotics, power systems, surveillance and reconnaissance systems, multimissile coordinated attacks, and intelligent transportation systems in [1-7]. In [8], based on the urgent needs of intelligent agriculture, the bioinspired coordination protocol was employed to achieve refinement in agricultural management. Consensus control is the most fundamental and most valuable core research of multiagent. The research directions include consensus control $[9,10]$, tracking control [11], aggregation control [12], formation control [13], and synchronous control $[1,14]$.

The consensus framework is proposed by Olfatil-Saber and Murray in $[14,15]$. Previous literature mainly focused on linear MASs and acquired large numbers of breakthrough achievements. Communication security, information samples, edge maps, and other related methods have also made some achievements in [16-18]. In practical mechanical systems, the problems of inherent nonlinearity and uncertainty would be further enhanced on account of the complex cooperative tasks, the limitation or lack of system dynamic cognition, and so forth. Several remarkable consensus control approaches of linear MASs would not directly be employed to nonlinear MASs. It is indispensable to apply adaptive technology and intelligent control methods to solve uncertain nonlinear distributed MASs.

Adaptive technology had become an effective method to address various nonlinear systems in the literature [19-21]. The neural networks (NNs) $[19,20]$ were related to the event trigger. The fuzzy logic systems (FLSs) [21-23] solved the finite time of stochastic nonlinear systems in [21]. Distributed nonlinear MASs among the literature [24-27] had been proved distinguished approximation ability to solve the 
unknown dynamics. The consensus control was discussed in [9], and consensus tracking control protocols were studied in [24-26]. The multileader consensus control method was researched in [27]. However, the above methods have not involved the problem of state time delays.

The differences in controller performance are often accompanied by state time delays which would reduce the speed of convergence, affect system performance, and lead to instability or ineffectiveness. Meanwhile, once time delays exceed the threshold, they would affect the whole systems. Therefore, time delays would be an important consideration. Some achievements had been made for time delays in uncertain nonlinear systems in [28-31]. Particularly, [30, 31] solved the time delayed switched systems. In [32, 33], LKFs were employed to eliminate time delays in cooperative tracking control with visual leader and leader-following, respectively. Nevertheless, it is obvious that the above omitted the constraints. In particular, it is unavoidable to consider the physical limits and safety performance of each agent, the mutual internal collision avoidance, and other operation restrictions. If ignoring constraints, system performance would be damaged or reduced and even would cause fatal accidents. From a practical point of view, the theoretical study of constraints becomes crucially important in MASs.

In the design of constrained controllers, reliable methods needed to be applied. The Barrier Lyapunov functions have been preferred as the primary candidate functions for designing controllers with constraints. The study in [34] presented the method to indirectly address the constraint by constructing barrier functions. Many BLFs, integral BLF (IBLF), and ABLF based adaptive approximate controllers were designed for different categories of the nonlinear systems in [34-41], and nonlinear MASs in [27, 42]. Integral barrier Lyapunov functions have been employed in [39-41]. The study in [27] proposed an adaptive fuzzy containment control based on the distributed observer and distributed sliding mode estimators with state constraints via multiple BLFs. Note that the above constraint strategies are indirect methods, which are applicable to the case that the feasibility of virtual controllers needs to be satisfied and the constraint boundaries are not infinitesimal. The nonlinear coordinate transformation function was used to construct the constraint completely dependent on states in [43] and [44], and the complicated feasibility analysis was reduced. The authors of $[45,46]$ proposed the control strategies to solve both the state constraints and state time-varying delays. Therefore, direct constraints on output or states are worth further investigation of the constrained cooperative control.

Motivated by the above analysis, this paper schedules a novel adaptive fuzzy distributed cooperative tracking control approach for a class of uncertain nonlinear MASs with state time-varying delays and constraints. The main contributions of the paper are as follows:

(1) Contrasting with the existing literature on time delays in $[32,33]$, time delays in the case of time variation are further investigated. The most important one is to overcome the failure of the decomposition theorem in nonstrict feedback. Meanwhile, state time-varying constraints are introduced into distributed time delayed MASs since the state time-varying delays and constraints further increase the difficulty of controller design, which is an enormous challenge.

(2) Distinguished from BLFs, the constraints construct by a nonlinear coordinate transformation in [43] that directly constrain on states, which are applicable to avoid complex feasibility analysis of virtual controllers. The constructed appropriate piecewise functions can effectively avoid the rapid growth and uncontrollability of the control rate and accelerate the convergence when the synchronization error is infinitely close to the origin.

The proposed strategy is aimed at the study of the timevarying state of the agents, which has great realistic significance and further enriches the exploration of the uncertain nonlinear MASs.

The organization of the article is as follows: the presentation and problem of the systems are described in Section 2; the adaptive fuzzy distributed cooperative control design is provided in Section 3; simulation is given in Section 4; and the conclusion is given in Section 5.

\section{Systems Presentation and Problem Description}

2.1. Systems Description and Problem Presentation. Consider a class of uncertain nonlinear distributed consensus systems with time-varying delay and the $i^{\text {th }}$ dynamic is described as

$$
\dot{x}_{i}(t)=f_{i}\left(x_{i}(t)\right)+\rho_{i}\left(x_{i}\left(t-\tau_{i}(t)\right)\right)+u_{i}(t),
$$

where $x_{i}(t)=\left[x_{i 1}, x_{i 2}, \ldots, x_{i m}\right]^{T} \in R^{m}$ represents the state vector of $i^{\text {th }}$ agent, $i=1,2, \ldots, n$, and $n$ is the number of agents; $f_{i}\left(x_{i}\right)$ and $\rho_{i}\left(x_{i}\left(t-\tau_{i}(t)\right)\right): R^{m} \longrightarrow R^{m}$ are unknown nonlinear continuous vector functions; $\tau_{i}(t)$ represents the uncertain time-varying delay; and $u_{i}(t) \in R^{m}$ is the control input vector.

Assumption 1. $\forall t \geq 0$, the $i^{\text {th }}$ unknown smooth nonlinear time delayed function $\rho_{i}\left(x_{i}\left(t-\tau_{i}(t)\right)\right)$, satisfies

$$
\left\|\rho_{i}\left(x_{i}\left(t-\tau_{i}(t)\right)\right)\right\| \leq q_{i}\left(x_{i}\left(t-\tau_{i}(t)\right)\right),
$$

where $q_{i}\left(x_{i}\left(t-\tau_{i}(t)\right)\right)$ is the known positive smooth function.

Remark 1. The unknown time-varying delays function $\rho\left(x_{i}\left(t-\tau_{i}(t)\right)\right)$ contains the time-varying delayed state. The separation technique is used to decompose the unknown with all the time-varying delays into positive continuous functions in order to compensate for the time delays by LKFs in [45].

Assumption 2. The unknown time-varying $\tau_{i}(t)$ should satisfy the following: (1) the $\dot{\tau}_{i}(t)$ is bounded by a known constant $\tau_{d \max }$ and $\dot{\tau}_{i}(t) \leq \tau_{d \max } \leq 1$ and (2) the continuous 
$\tau_{i}(t)$ should be uniformly bounded by a known constant $\tau_{\max }$ and $\tau_{i}(t) \leq \tau_{\max }$ in $[33,45,46]$.

The desired reference leader signal is described by the following dynamic function:

$$
\dot{x}_{l}(t)=g_{l}(t)
$$

where $x_{l}(t) \in R^{m}$ is the state of reference leader and $g_{l}(t) \in R^{m}$ is a bounded smooth vector function.

The time-varying state constraint is considered, and the constraint boundary of $i^{\text {th }}$ agent meets requirements as follows:

$$
-k_{c_{j}}(t)<x_{i j}(t)<k_{c_{j}}(t),
$$

where $x_{i j}$ represents the $j^{\text {th }}$ state of the $i^{\text {th }}$ agent; $j=1,2, \ldots, m ; k_{c_{j}}(t)$ is a user-defined time-varying bound function in $j^{\text {th }}$ state; and $k_{c}(t) \in R^{m}$. The initial value should satisfy $-k_{c_{j}}(0)<x_{i j}(0)<k_{c_{j}}(0)$.

The requirement of (4) can be satisfied by choosing a nonlinear coordinate transformation as follows:

$$
s_{i j}(t)=\frac{x_{i j}(t)}{\left(k_{c_{j}}(t)+x_{i j}(t)\right)\left(k_{c_{j}}(t)-x_{i j}(t)\right)},
$$

where $s_{i j}(t)$ is a constructed state transition that converts the original $x_{i j}(t)$ bounded within $\left(-k_{c_{j}}(t), k_{c_{j}}(t)\right)$ into a nonconstrained state, $s_{i}(t)=\left[s_{i 1}, s_{i 2}, \ldots, s_{i m}\right]^{T} \stackrel{c_{j}}{\epsilon} R^{m}$.

Then, (5) satisfies

$$
\left\{\begin{array}{c}
\lim _{x_{i}(t) \longrightarrow-k_{c}(t)} s_{i}(t)=-\infty \\
\lim _{x_{i}(t) \longrightarrow k_{c}(t)} s_{i}(t)=+\infty .
\end{array}\right.
$$

Take the derivative of $s_{i j}(t)$ as follows:

$$
\dot{s}_{i j}(t)=\frac{\partial s_{i j}(t)}{\partial x_{i j}(t)} \dot{x}_{i j}(t)+\frac{\partial s_{i j}(t)}{\partial k_{c_{j}}(t)} \dot{k}_{c_{j}}(t),
$$

where $\partial s_{i j}(t) / \partial x_{i j}(t)=k_{c_{j}}^{2}(t)+x_{i j}^{2}(t) /\left(k_{c_{j}}^{2}(t)-x_{i j}^{2}(t)\right)^{2}$ and $\partial s_{i j}(t) / \partial k_{c_{j}}(t)=-2 x_{i j}(t) k_{c_{j}}(t) /\left(k_{c_{j}}^{2}(t)-x_{i j}^{2}(t)\right)^{2}$.

The state of the leader in (3) can be transformed by the same form of (5); it can be expressed as

$$
\alpha_{l j}(t)=\frac{x_{l j}(t)}{\left(k_{c_{j}}(t)+x_{l j}(t)\right)\left(k_{c_{j}}(t)-x_{l j}(t)\right)},
$$

where $\alpha_{l j}(t)$ is a state transition to transform the constrained $x_{l j}(t)$ within $\left(-k_{c_{j}}(t), k_{c_{j}}(t)\right)$ into an unconstrained state, $\alpha_{i}(t)=\left[\alpha_{i 1}, \alpha_{i 2}, \ldots, \alpha_{i m}\right]^{T} \in R^{m}$. Then, (8) satisfies

$$
\left\{\begin{array}{c}
\lim _{x_{l}(t) \longrightarrow-k_{c}(t)} \alpha_{l}(t)=-\infty, \\
\lim _{x_{l}(t) \longrightarrow k_{c}(t)} \alpha_{l}(t)=+\infty .
\end{array}\right.
$$

Take the derivative of (8) as follows:

$$
\dot{\alpha}_{l j}(t)=\frac{\partial \alpha_{l j}(t)}{\partial x_{l j}(t)} \dot{x}_{l j}(t)+\frac{\partial \alpha_{l j}(t)}{\partial k_{c_{j}}(t)} \dot{k}_{c_{j}}(t),
$$

where $\quad \partial \alpha_{l j}(t) / \partial x_{l j}(t)=k_{c_{j}}^{2}(t)+x_{l j}^{2}(t) /\left(k_{c_{j}}^{2}(t)-x_{l j}^{2}(t)\right)^{2}$ and $\partial \alpha_{l j}(t) / \partial k_{c_{j}}(t)=-2 x_{l j}(t) k_{c_{j}}(t) /\left(k_{c_{j}}^{2}(t)-x_{l j}^{2}(t)\right)^{2}$.
Remark 2. The states $x_{i}$ and $x_{l}$ are converted into $s_{i}$ and $\alpha_{l}$, respectively; $\partial s_{i j}(t) / \partial x_{i j}(t)>0$ and $\partial \alpha_{l j}(t) / \partial x_{l j}(t)>0$ are invertible functions; and $s_{i j}(t)$ and $\alpha_{l j}(t)$ are monotonically increasing. Just proving $s_{i j}(t)$ and $\alpha_{l j}(t)$ are bounded, by criteria of monotone bounded, the original states $x_{i}$ and $x_{l}$ would converge and satisfy the constraint region.

Assumption 3. The time-varying bound vector functions $k_{c}(t)$ and $\dot{k}_{c}(t)$ satisfy the following: $\forall t \geq 0$; there exist positive constant vectors $K_{c} \in R^{m}$ and $K_{c}^{1} \in R^{m}$ that satisfy $\left|k_{c_{j}}(t)\right| \leq K_{c_{j}}$ and its time derivative $\dot{k}_{c_{j}}(t)$ satisfies $\left|\dot{k}_{c_{j}}(t)\right| \leq K_{c_{j}}^{1_{j}}$.

Remark 3. In numbers of practical cooperative control of multiagent systems, inevitably, constraints need to be addressed due to physical limitations or performance. If the constraints were ignored, the systems may be degraded or damaged by interagent influences, and even fatal accidents would occur. State constraints are more effective in solving states of transient or steady real systems than only with output constraints. The control process is timevarying, so it is significant to study the time-varying full state constraints.

Remark 4. The constraints based on BLFs (or IBLFs) require additional complex stability analysis to ensure the feasibility conditions of virtual controllers. The state-dependent transition method in $[43,44]$ which can circumvent the above problems, flexibly solve initial conditions, and reduce the difficulty of stability analysis.

Remark 5. The nonlinear coordinate transformation function is constructed and purely dependent on the constraint states. Introducing a new coordinate transformation for the backstepping design of MASs, it directly responses to state constraints and completely avoids the harsh feasibility conditions. This will get rid of the tedious feasibility verification. Let the designer have more freedom and be humanized to choose the designed parameters in implementations. Apply a wider range of initial conditions.

This paper only shows the feasibility and effectiveness of constructing state time-varying constraints to distributed nonlinear and uncertain time-varying delayed MASs. The asymmetric forms would be further discussed in other papers.

2.1.1. Control Objective. Devise an adaptive fuzzy decentralized tracking controller $u_{i}$ that, for the dynamic of agents described in (1) and (3), can guarantee the following:

(i) All followers converge to the desired small neighborhood around the origin by tracking the leader. (ii) The full states would not violate the time-varying constraint boundaries in (5) and (8). (iii) All the signals in the closedloop systems are SUUB.

The following assumptions and lemmas should be satisfied in order to establish a cooperative control objective.

2.2. Algebraic Graph Theory and Notations. An undirected connected topology expresses as $G=(V, E, A)$, where 
$V=\left\{v_{1}, v_{2}, \ldots, v_{n}\right\}$ denotes a set of nodes and $v_{i}$ is $i^{\text {th }}$ node. $E \subseteq V \times V$ denotes the set of edges, $\wp_{i j}=\left(v_{i}, v_{j}\right) \in E$ refers to the edge from node $i$ to node $j$, and $\wp_{i j}=\wp_{j i}$. $A=\left[a_{i j}\right] \in R^{n \times n}$ is a weighted adjacency matrix that a weight corresponds to the edge, and when $\wp_{i j} \in R^{n \times n}, a_{i j}=1$. Otherwise, $a_{i j}=0$. In particular, define $a_{i i}=0$.

The graph Laplacian matrix $L$ is given in the following definition:

$$
L=D-A \text {, }
$$

where $\quad L=\left[l_{i j}\right] \subset R^{n \times n} ; \quad D=\operatorname{diag}\left\{d_{1}, d_{2}, \ldots, d_{n}\right\} \in R^{n \times n}$ defines an in-degree matrix; $d_{i}=\sum_{j \in N_{i}} a_{i j}$ represents the in-degree of node $i ; N_{i}=\{j:(j, i) \in E\} ; L$ is an irreducible matrix; and $[1,1, \ldots, 1]^{T} \in R^{n}$ is a right eigenvector with the eigenvalue zero.

Lemma 1. The graph Laplacian matrix $L$ is a semidefinite symmetric matrix; all eigenvalues are nonnegative real; and its $n$ real eigenvalues can be listed in ascending order as follows:

$$
0=\lambda_{1}<\lambda_{2} \leq \lambda_{3} \leq \ldots \leq \lambda_{n} \leq C,
$$

where $C=2\left(\max _{1 \leq i \leq n} d_{i}\right)$ is algebraic connectivity and it is used to analyze the rate of consensus convergence.

Lemma 2. $\forall t>0$, the continuous function $V(t)$ satisfies $V(t)>0$, and $V(0)$ is bounded. If it can prove the inequality $\dot{V}(t) \leq-\ell V(t)+\hbar$ where $\ell>0, \hbar>0$, then

$$
\dot{V}(t) \leq V(0) e^{-\ell t}+\frac{\hbar}{\ell}\left(1-e^{-\ell t}\right) .
$$

Assumption 4. In the graph topology $G$, for the leader, there is at least one directed path from the root node to follower nodes.

2.3. Distributed Controller. The proposed distributed control algorithm can make arbitrary $i^{\text {th }}$ agent feedback to itself and communicate with neighbor agents via adjacent matrix. Since fixed topology graphs are the basis for different types of switching connection topology and are widely applied such as $[27,28]$, this paper is based on the fixed topological form.

The synchronization error $e_{i}(t) \in R^{m}$ consists of the $i^{\text {th }}$ local consensus error and the tracking error among the leader and followers, and that can be defined as follows:

$$
e_{i}(t)=\sum_{j=1}^{N_{i}} a_{i j}\left(s_{i}(t)-s_{j}(t)\right)+b_{i}\left(s_{i}(t)-\alpha_{l}(t)\right),
$$

where $a_{i j}$ represents the element in adjacency matrix $A . b_{i}$ is the communication weight when only $i^{\text {th }}$ agent exchanges information with leader $b_{i}>0$; otherwise $b_{i}=0$, and $B=$ $\operatorname{diag}\left\{b_{1}, b_{2}, \ldots, b_{n}\right\}$ is the communication weight matrix and satisfies $b_{1}+b_{2}+\cdots+b_{n}>0$ :

Let

$$
\widetilde{L}=L+B,
$$

where $\widetilde{L}$ is a symmetrical positive definite matrix and its real eigenvalues $\widetilde{\lambda}_{1}, \widetilde{\lambda}_{2}, \ldots, \widetilde{\lambda}_{n}$ satisfy Lemma 1 .

Based on the matrix theory and graph theory, it can be readily concluded that zero is an $m$-multiplicity eigenvalue of $\widetilde{L} \otimes I_{m} ; \otimes$ and $I_{m}$, respectively, stand for the Kronecker product and identity matrix of dimension $m \times m$. Let $\varphi_{11}, \varphi_{12}, \ldots, \varphi_{1 m}, \ldots, \varphi_{2 m}, \ldots, \varphi_{n m}$ be the eigenvectors and $\hat{\lambda}_{2}, \ldots, \widetilde{\lambda}_{n}$ eigenvalues of $\tilde{L} \otimes I_{m}$ such there can be a set of orthogonal bases of $R^{\mathrm{nm}}$. The definition is given by graph theory:

$$
\widetilde{L} \otimes I_{m}=P \Lambda P^{T},
$$

where $P^{T} P=\mathrm{PP}^{T}=I_{n m}, I_{n m}$ denoting identity matrix of dimension $n m \times n m$ and $P=\left[\varphi_{11}, \varphi_{12}, \ldots \varphi_{\mathrm{nm}}\right] \in R^{n m \times n m}$.

Define the tracking error vector as the following form:

$$
\varsigma_{i}(t)=s_{i}(t)-\alpha_{l}(t),
$$

where $\varsigma_{i}(t)=\left[\varsigma_{i 1}(t), \varsigma_{i 2}(t), \ldots, \varsigma_{i m}(t)\right]^{T} \in R^{m} \quad$ represents the tracking error between the $i^{\text {th }}$ follower agent and the leader agent. Then, (14) can be rewritten as

$$
e_{i}(t)=\sum_{j=1}^{N_{i}} a_{i j}\left(\varsigma_{i}(t)-\varsigma_{j}(t)\right)+b_{i} \varsigma_{i}(t),
$$

where $e_{i}(t)=\left[e_{i 1}(t), e_{i 2}(t), \ldots, e_{i m}(t)\right]^{T}$.

The unknown functions and variables in multiagent systems can be approximated by FLSs as follows:

$$
F_{i j}\left(X_{i j}\right)=\omega_{i j}^{T} \Psi_{i j}\left(X_{i j}\right)+\varepsilon_{i j}
$$

where $\omega_{i j} \in R^{l_{j}}$ is the optimal fuzzy weight vector, $l_{j}$ denotes the number of fuzzy rules, $\Psi_{i j}\left(X_{i j}\right)$ is the fuzzy basis vector function, $\varepsilon_{i j}$ shows estimation error satisfied $\left\|\varepsilon_{i j}\right\| \leq \bar{\varepsilon}_{i j}$, and $\bar{\varepsilon}_{i j}$ is an arbitrary indeterminate positive constant.

\section{Adaptive Fuzzy Distributed Cooperative Control Design}

In this section, an adaptive fuzzy distributed cooperative control algorithm will be designed for (1) and (3). Define the following candidate function:

$$
V_{\omega}=\frac{1}{2} \varsigma(t)^{T} Q \varsigma(t)+\frac{1}{2} \sum_{i=1}^{n} \operatorname{tr}\left[\widetilde{\omega}_{i}^{T} \Gamma_{i}^{-1} \widetilde{\omega}_{i}\right],
$$

where $\varsigma(t)=\left(\varsigma_{1}^{T}(t), \varsigma_{2}^{T}(t), \ldots, \varsigma_{n}^{T}(t)\right)^{T} \in R^{\mathrm{nm}}$ is the consensus error vector that $\varsigma_{i}(t)$ has defined in (17), then $\varsigma(t)=\left(\widetilde{L} \otimes I_{m}\right) e(t), Q=P \hat{\Lambda}^{-1} P^{T}, \widetilde{\omega}_{i j}=\omega_{i j}-\widehat{\omega}_{i j}, \widehat{\omega}_{i j}$ is the estimate of adaptive laws, and $\omega_{i j}$ and $\widehat{\omega}_{i j}$ will be given later.

The inequality can be described as follows:

$$
\frac{\lambda_{\min }(Q)}{2} \leq \frac{1}{2} \varsigma(t)^{T} Q \varsigma(t) \leq \frac{\lambda_{\max }(Q)}{2}
$$

where $\lambda_{\min }(Q)$ and $\lambda_{\max }(Q)$ are the smallest and largest eigenvalues of $Q$, respectively. From (16), it yields 


$$
\begin{aligned}
\frac{1}{2}\left(\varsigma(t)^{T} Q \varsigma(t)\right) & =\frac{1}{2} e^{T}(t)\left(\widetilde{L} \otimes I_{m}\right)^{T} P \widehat{\Lambda}^{-1} P^{T}\left(\widetilde{L} \otimes I_{m}\right) e(t)=\frac{1}{2} e^{T}(t) P \Lambda P^{T} P \widehat{\Lambda}^{-1} P^{T} P \Lambda P^{T} e(t) \\
& =\frac{1}{2} e^{T}(t)\left(\widetilde{L} \otimes I_{m}\right) e(t)=\frac{1}{2} \varsigma(t)^{T} e(t)=\frac{1}{2} \sum_{i=1}^{n} \varsigma_{i}^{T}(t) e_{i}(t),
\end{aligned}
$$

where $\Lambda=\operatorname{diag}\left\{0 I_{m}, \widetilde{\lambda}_{2} I_{m}, \ldots, \widetilde{\lambda}_{n} I_{m}\right\}$.

Redefine the Lyapunov candidate function as follows:

$$
V_{\omega}=\frac{1}{2} \sum_{i=1}^{n} \varsigma_{i}^{T}(t) e_{i}(t)+\frac{1}{2} \sum_{i=1}^{n} \operatorname{tr}\left[\widetilde{\omega}_{i}^{T} \Gamma_{i}^{-1} \widetilde{\omega}_{i}\right]
$$

$$
\dot{V}_{\omega}=\frac{1}{2} \dot{\zeta}(t)^{T} Q \varsigma(t)+\frac{1}{2} \varsigma(t)^{T} Q \dot{\zeta}(t)+\frac{1}{2} \sum_{i=1}^{n} \operatorname{tr}\left[\dot{\tilde{\omega}}_{i}^{T} \Gamma_{i}^{-1} \widetilde{\omega}_{i}\right]+\frac{1}{2} \sum_{i=1}^{n} \operatorname{tr}\left[\widetilde{\omega}_{i}^{T} \Gamma_{i}^{-1} \dot{\tilde{\omega}}_{i}\right]=\sum_{i=1}^{n} e_{i}^{T}(t) \dot{\zeta}_{i}(t)-\sum_{i=1}^{n} \operatorname{tr}\left[\widetilde{\omega}_{i}^{T} \Gamma_{i}^{-1} \dot{\hat{\omega}}_{i}\right] .
$$

$\dot{V}_{\omega}$ can be written as follows:

$$
\dot{V}_{\omega}=\sum_{j=1}^{m} \dot{V}_{\omega j}=\sum_{j=1}^{m} \sum_{i=1}^{n}\left[e_{i j}^{T}(t) \dot{\zeta}_{i j}(t)-\widetilde{\omega}_{i j}^{T} \Gamma_{i j}^{-1} \dot{\hat{\omega}}_{i j}\right]
$$

The derivative of $\varsigma_{i j}(t)$ in the form of (17) can be described as

$$
\dot{V}_{\omega j} \leq \sum_{i=1}^{n} e_{i j}^{T}(t)\left\{\frac{\partial s_{i j}(t)}{\partial x_{i j}(t)}\left[u_{i j}(t)+\rho_{i j}\left(x_{i j}\left(t-\tau_{i}(t)\right)\right)+f_{i j}\left(x_{i j}(t)\right)\right]+K_{i j}(t)-\frac{\partial \alpha_{l j}(t)}{\partial x_{l j}(t)} g_{l j}(t)\right\}-\sum_{i=1}^{n}\left(\widetilde{\omega}_{i j}^{T} \Gamma_{i j}^{-1} \dot{\hat{\omega}}_{i j}\right),
$$

where $K_{i j}(t)$ is $i^{\text {th }}$ time-varying gain described as follows:

$$
K_{i j}(t)=\sup \sqrt{\left(\frac{\partial s_{i j}(t)}{\partial k_{c_{j}}(t)} \dot{k}_{c_{j}}(t)\right)^{2}+\left(\frac{\partial \alpha_{l_{j}}(t)}{\partial k_{c_{j}}(t)} \dot{k}_{c_{j}}(t)\right)^{2}+\beta_{i j}}
$$

$\beta_{i j}$ is a positive parameter to be given.

Using Young's inequality and Assumption 1, in order to separate $e_{i j}$ and uncertainties $\rho_{i j}\left(x_{i j}\left(t-\tau_{i}(t)\right)\right)$, the inequality gets

$$
e_{i j}^{T}(t) \frac{\partial s_{i j}(t)}{\partial x_{i j}(t)} \rho_{i j}\left(x_{i j}\left(t-\tau_{i}(t)\right)\right) \leq \frac{1}{2 \sqrt{v}}\left\|e_{i j}(t)\right\|^{2} M_{i j}^{2}+\frac{1}{2} v q_{i j}^{2}\left(x_{i j}\left(t-\tau_{i}(t)\right)\right)
$$

where $v=1-\tau_{d \max }$ has been illustrated in Remark 4 and $M_{i j}=\left\|\partial s_{i j}(t) / \partial x_{i j}(t)\right\|$.

The unknown smooth function $F_{i j}\left(X_{i j}\right)$ is approximated by FLSs to tackle the unknown dynamics functions and parameters. $F_{i j}\left(X_{i j}\right)$ is denoted as

$$
F_{i j}\left(X_{i j}\right)=f_{i j}\left(x_{i j}(t)\right)+\frac{1}{2} \frac{1}{\sqrt{v}} e_{i j}(t) \frac{\partial s_{i j}(t)}{\partial x_{i j}(t)},
$$

where $X_{i 1}=\left[x_{i 1}, x_{i 2}, k_{c_{1}}, e_{i 1}\right]^{T}, X_{i 2}=\left[x_{i 1}, x_{i 2}, k_{c_{2}}, e_{i 2}\right]^{T}$, and $\left\|e_{i j}(t)\right\|^{2} M_{i j}^{2}$ takes the norm of $e_{i j}(t) \partial s_{i j}(t) / \partial x_{i j}(t)$ in (29).

Equation (30) can be estimated by (19) as follows:

$$
F_{i j}\left(X_{i j}\right)=\omega_{i j}^{T} \Psi_{i j}\left(X_{i j}\right)+\varepsilon_{i j}
$$
yield

Combining (28), (29), and (30) by FLSs, then (27) can 


$$
\dot{V}_{\omega j} \leq \sum_{i=1}^{n} e_{i j}^{T}(t)\left[\frac{\partial s_{i j}(t)}{\partial x_{i j}(t)}\left(u_{i j}(t)+\omega_{i j}^{T} \Psi_{i j}\left(X_{i j}\right)+\varepsilon_{i j}\right)+U_{i j}(t)\right]+\frac{1}{2} \sum_{i=1}^{n} v q_{i j}^{2}\left(x_{i j}\left(t-\tau_{i}(t)\right)\right)-\sum_{i=1}^{n}\left(\widetilde{\omega}_{i j}^{T} \Gamma_{i j}^{-1} \dot{\hat{\omega}}_{i j}\right)
$$

where $U_{i j}(t)=K_{i j}(t)-\partial \alpha_{l j}(t) / \partial x_{l j}(t) g_{l j}(t)$.

By using Young's inequality, we obtain the following:

$$
\sum_{i=1}^{n} e_{i j}^{T}(t) \frac{\partial s_{i j}(t)}{\partial x_{i j}(t)} \varepsilon_{i j} \leq \frac{1}{2} \sum_{i=1}^{n}\left\|e_{i j}(t)\right\|^{2} M_{i j}^{2}+\frac{1}{2} \sum_{i=1}^{n} \bar{\varepsilon}_{i j}^{2}
$$

Combining (33) into (32), we obtain

$$
\begin{aligned}
\dot{V}_{\omega j} \leq & \sum_{i=1}^{n} e_{i j}^{T}(t)\left[\frac{\partial s_{i j}(t)}{\partial x_{i j}(t)}\left(u_{i j}(t)+\omega_{i j}^{T} \Psi_{i j}\left(X_{i j}\right)\right)+U_{i j}(t)\right]+\frac{1}{2} \sum_{i=1}^{n} v q_{i j}^{2}\left(x_{i j}\left(t-\tau_{i}(t)\right)\right) \\
& -\sum_{i=1}^{n}\left(\widetilde{\omega}_{i j}^{T} \Gamma_{i j}^{-1} \dot{\hat{\omega}}_{i j}\right)+\frac{1}{2} \sum_{i=1}^{n}\left\|e_{i j}(t)\right\|^{2} M_{i j}^{2}+\sum_{i=1}^{n} \frac{1}{2} \bar{\varepsilon}_{i j}^{2} .
\end{aligned}
$$

The Lyapunov-Krasovskii function is constructed to compensate uncertain time-varying delays as follows:

$$
V_{\rho}(t)=\sum_{i=1}^{m} V_{\rho j}(t)=\frac{1}{2} \sum_{i=1}^{m} \sum_{i=1}^{n} \int_{t-\tau_{i}(t)}^{t} \beta_{i j}\left(x_{i j}(s)\right) d s,
$$

where $\beta_{i j}\left(x_{i j}(s)\right) \leq q_{i j}^{2}\left(x_{i j}(s)\right)$ has been defined under Assumption 1 .
Take the derivative of $V_{\rho j}(t)$. It obtains

$$
\dot{V}_{\rho j}(t) \leq \frac{1}{2} \sum_{i=1}^{n} q_{i j}^{2}\left(x_{i j}(t)\right)-\frac{1}{2} v \sum_{i=1}^{n} q_{i j}^{2}\left(x_{i j}\left(t-\tau_{i}(t)\right)\right)
$$

The Lyapunov function candidate is chosen by combining (23) and (35) as follows:

$$
\begin{aligned}
V(t) & =V_{\omega}+V_{\rho}(t) \\
\sum_{j=1}^{m} V_{i j}(t) & =\sum_{j=1}^{m} V_{\omega j}+\sum_{i=1}^{m} V_{\rho j}(t), \\
\sum_{j=1}^{m} V_{i j}(t) & =\sum_{j=1}^{m}\left(\frac{1}{2} \sum_{i=1}^{n} \varsigma_{i j}^{T}(t) e_{i j}(t)+\frac{1}{2} \sum_{i=1}^{n}\left(\widetilde{\omega}_{i j}^{T} \Gamma_{i j}^{-1} \widetilde{\omega}_{i j}\right)\right)+\frac{1}{2} \sum_{j=1}^{m} \sum_{i=1}^{n} \int_{t-\tau_{i}(t)}^{t} \beta_{i j}\left(x_{i j}(s)\right) d s .
\end{aligned}
$$
obtain

According to (34) and (36), the derivative of $V_{i j}(t)$ can

$$
\dot{V}_{i j} \leq \sum_{i=1}^{n} e_{i j}^{T}(t)\left[\frac{\partial s_{i j}(t)}{\partial x_{i j}(t)}\left(u_{i j}(t)+\omega_{i j}^{T} \Psi_{i j}\left(X_{i j}\right)\right)+U_{i j}(t)\right]+\frac{1}{2} \sum_{i=1}^{n} q_{i j}^{2}\left(x_{i j}(t)\right)-\sum_{i=1}^{n}\left(\widetilde{\omega}_{i j}^{T} \Gamma_{i j}^{-1} \dot{\widehat{\omega}}_{i j}\right)+\frac{1}{2} \sum_{i=1}^{n}\left\|e_{i j}(t)\right\|^{2} M_{i j}^{2}+\frac{1}{2} \sum_{i=1}^{n} \bar{\varepsilon}_{i j}^{2} .
$$

The designed decentralized controller has been described as follows:

$$
u_{i j}(t)= \begin{cases}\left(\frac{\partial s_{i j}(t)}{\partial x_{i j}(t)}\right)^{-1}\left[-k_{i j}(t) e_{i j}(t)-\widehat{\omega}_{i j}^{T} \Psi_{i j}\left(X_{i j}\right)\right. & \\ \left.-\frac{1}{2} \frac{\left\|e_{i j}(t)\right\| q_{i j}^{2}\left(x_{i j}(t)\right)}{\left\|e_{i j}(t)\right\|^{2}+\chi_{i j}}-U_{i j}(t)\right], & e_{i j}(t) \in \Omega_{\delta_{i}}^{c}, \\ 0, & e_{i j}(t) \in \Omega_{\delta_{i}},\end{cases}
$$


where $\partial s_{i j}(t) / \partial x_{i j}(t)$ is a monotonically increasing function and the inverse must exist in Remark $1 . k_{i j}(t)$ is the controller gain that will be defined later. $\Omega_{\delta_{i}}=\left\{e_{i j}(t) \mid\left\|e_{i j}(t)\right\|<\delta_{i}\right\}$ is a compact set, $\Omega_{\delta_{i}}^{c}$ is its complement set, $\delta_{i}$ is an arbitrarily positive real number, and its definition will be given later. Once in $\Omega_{\delta_{i}}$ near the origin, there is no need to impose on control. The piecewise function $\chi_{i j}$ satisfies the following:

$$
\chi_{i j}= \begin{cases}1, & e_{i j}(t) \in \Omega_{\delta_{i}}^{c}, \\ 0, & e_{i j}(t) \in \Omega_{\delta_{i}} .\end{cases}
$$

Remark 6. Particularly, singularities may occur when $e_{i}(t)=$ $[0]_{m}$ in cooperative control strategy in $1 / 2\left\|e_{i j}(t)\right\|^{-1}$ $q_{i j}^{2}\left(x_{i j}(t)\right)$. The function $\lim _{e_{i j}(t) \longrightarrow 0} e_{i j}^{-1}(t)=\infty$ means the limit of synchronization error function infinitely approaches the sphere near the origin. Even though it is not equal to zero and there is no singular value, it is still uncontrollable. Therefore, (40) is designed to prevent the occurrence and accelerate the convergence rate.

Figure 1 is the flow diagram of the control approach for a class of uncertain nonlinear MASs with state time-varying constraints and delays, which can clearly guide the construction process of the control strategy.

From Remark 3, the adaptive law for $\widehat{\omega}_{i j}$ is designed as follows:

$$
\dot{\hat{\omega}}_{i j}=\Gamma_{i j}\left\{e_{i j}(t) \Psi_{i j}\left(X_{i j}\right)-\kappa_{i j} \widehat{\omega}_{i j}\right\},
$$

where $\Gamma_{i j}$ is a positive symmetric gain. $\kappa_{i j}$ is the positive constant to improve the approximation error robustness of FLSs.
When $e_{i j}(t) \in \Omega_{\delta_{i}}^{c}$, combining (39), (40), and (41) into (38) yields

$$
\dot{V}_{i j}(t) \leq \sum_{i=1}^{n}-k_{i j}(t)\left\|e_{i j}(t)\right\|^{2}+\sum_{i=1}^{n} \kappa_{i j} \widetilde{\omega}_{i j}^{T} \widehat{\omega}_{i j}+\frac{1}{2} \sum_{i=1}^{n}\left\|e_{i j}(t)\right\|^{2} M_{i j}^{2}+\frac{1}{2} \sum_{i=1}^{n} \bar{\varepsilon}_{i j}^{2} .
$$

Using Young's inequality, it follows that

$$
\widetilde{\omega}_{i j}^{T} \widehat{\omega}_{i j}=\widetilde{\omega}_{i j}^{T}\left(\omega_{i j}-\widetilde{\omega}_{i j}\right) \leq \frac{1}{2} \omega_{i j}^{2}-\frac{1}{2} \widetilde{\omega}_{i j}^{T} \widetilde{\omega}_{i j} .
$$

According to (43) into (42), then $\dot{V}_{i j}(t)$ obtains

$$
\dot{V}_{i j}(t) \leq \sum_{i=1}^{n}\left[-k_{i j}(t)\left\|e_{i j}(t)\right\|^{2}+\frac{1}{2} \sum_{i=1}^{n} M_{i j}^{2}\left\|e_{i j}(t)\right\|^{2}\right]-\frac{1}{2} \kappa_{i j} \widetilde{\omega}_{i j}^{T} \widetilde{\omega}_{i j}+\eta_{j 2},
$$

where $\eta_{j 2}=1 / 2 \sum_{i=1}^{n} \kappa_{i j} \omega_{i j}^{2}+1 / 2 \sum_{i=1}^{n} \bar{\varepsilon}_{i j}^{2}$

The definition of controller gain $k_{i j}(t)$ is described briefly as follows:

$$
k_{i j}(t)=k_{i j}^{0}+k_{i j}^{1}(t),
$$

where $k_{i j}^{0} \geq 1 / 2 M_{i j}^{2}$, and $k_{i j}^{1}(t)$ satisfies

$$
k_{i j}^{1}(t)=\frac{\gamma_{i j}}{2}\left[\lambda_{\max }(Q)+\frac{1}{\left\|e_{i j}(t)\right\|^{2}+\chi_{i j}} \int_{t-\tau_{\max }}^{t} \beta_{i j}\left(x_{i j}(s)\right) d s\right] .
$$

Substitute (45) and (46) into (44); it yields

$$
\dot{V}_{i j}(t) \leq-\frac{1}{2} \sum_{i=1}^{n} \gamma_{i j} \lambda_{\max }(Q)\left\|e_{i j}(t)\right\|^{2}-\frac{1}{2} \sum_{i=1}^{n} \frac{\kappa_{i j}}{\Gamma_{i j}^{-1}}\left(\widetilde{\omega}_{i j}^{T} \Gamma_{i j}^{-1} \widetilde{\omega}_{i j}\right)-\frac{1}{2} \sum_{i=1}^{n} \gamma_{i j} \int_{t-\tau_{\max }}^{t} \beta_{i j}\left(x_{i j}(s)\right) d s+\eta_{j 2},
$$

where $\beta_{i j}\left(x_{i j}(s)\right)>0$ has been defined; therefore, the integral inequality satisfies

By $(48), \dot{V}_{i j}(t)$ can obtain

$$
\frac{1}{2} \sum_{i=1}^{n} \int_{t-\tau_{\max }}^{t} \beta_{i j}\left(x_{i j}(s)\right) d s \geq \frac{1}{2} \sum_{i=1}^{n} \int_{t-\tau_{i}(t)}^{t} \beta_{i j}\left(x_{i j}(s)\right) d s .
$$

$$
\dot{V}_{i j}(t) \leq-\frac{1}{2} \sum_{i=1}^{n} \gamma_{i j} \lambda_{\max }(Q)\left\|e_{i j}(t)\right\|^{2}-\frac{1}{2} \sum_{i=1}^{n} \frac{\kappa_{i j}}{\Gamma_{i j}^{-1}}\left(\widetilde{\omega}_{i j}^{T} \Gamma_{i j}^{-1} \widetilde{\omega}_{i j}\right)-\frac{1}{2} \sum_{i=1}^{n} \gamma_{i j} \int_{t-\tau_{i}(t)}^{t} \beta_{i j}\left(x_{i j}(s)\right) d s+\eta_{j 2} .
$$




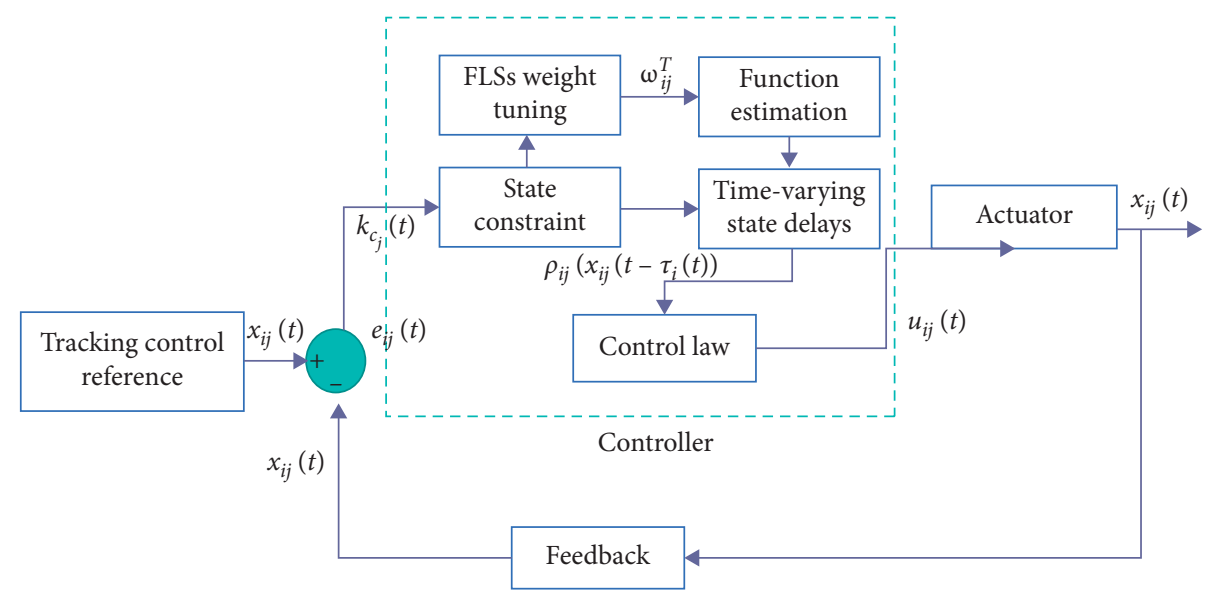

Figure 1: The block diagram illustrating the flow of the proposed control approach.

From (21), $\dot{V}_{i j}(t)$ yields

$$
\dot{V}_{i j}(t) \leq-\eta_{j 1} V_{i j}(t)+\eta_{j 2}
$$

where $\eta_{j 1}=\min _{1 \leq i \leq n}\left\{\gamma_{i j}, \quad \kappa_{i j} / \Gamma_{i j}^{-1}\right.$.

Then, compute this sum and integral of $\dot{V}_{i j}(t)$ obtained that

$$
V(t) \leq V(0) e^{-\eta_{1} t}+\left[\frac{\eta_{2}}{\eta_{1}}\left(1-e^{-\eta_{1} t}\right)\right]
$$

where $\eta_{1}=\min _{1 \leq j \leq m} \eta_{j 1}, \eta_{2}=\sum_{j=1}^{m} \eta_{j 2}$.

Theorem 1. For nonlinear dynamics (1) and (3) with Assumptions 1-3 and the controller gains in (39), the adaptive law is given by (41). By selecting appropriate parameters, the adaptive fuzzy cooperative control strategy can guarantee the following:

(1) The synchronization error converges to the desired small neighborhood near the origin

(2) The state time-varying constraints for agents given by (5) and (8) would not be violated

(3) The time-varying delays can be compensated by LKFs. The signals in the closed-loop systems can be verified SUUB

Proof. When $e_{i j}(t) \in \Omega_{\delta_{i}}^{c}$, choosing the Lyapunov function in (37), the derivative of $V(t)$ is as follows:

$$
\begin{aligned}
\dot{V}(t) \leq & \sum_{j=1}^{m}\left\{\sum_{i=1}^{n} \bar{e}_{i j}^{T}(t)\left\{\frac{\partial s_{i j}(t)}{\partial x_{i j}(t)}\left[u_{i j}(t)+\rho_{i j}\left(x_{i j}\left(t-\tau_{i}(t)\right)+f_{i j}\left(x_{i j} t\right)\right)\right]+U_{i j}(t)\right\}\right. \\
& \left.-\sum_{i=1}^{n}\left(\widetilde{\omega}_{i j}^{T} \Gamma_{i j}^{-1} \dot{\widehat{\omega}}_{i j}\right)+\sum_{i=1}^{n} q_{i}^{2}\left(x_{i}(t)\right)-\frac{1}{2} v \sum_{i=1}^{n} q_{i}^{2}\left(x_{i}\left(t-\tau_{i}(t)\right)\right)\right\} .
\end{aligned}
$$

Applying the results in (28), (29), (30), (33), and (36) into (52), it obtains

$$
\dot{V}(t) \leq \sum_{j=1}^{m}\left\{\sum_{i=1}^{n} e_{i j}^{T}(t)\left[\frac{\partial s_{i j}(t)}{\partial x_{i j}(t)}\left(u_{i j}(t)+\omega_{i j}^{T} \Psi_{i j}\left(X_{i j}\right)\right)+U_{i j}(t)\right]+\sum_{i=1}^{n} \frac{1}{2} q_{i j}^{2}\left(x_{i j}(t)\right)-\sum_{i=1}^{n}\left(\widetilde{\omega}_{i j}^{T} \Gamma_{i j}^{-1} \dot{\hat{\omega}}_{i j}\right)+\frac{1}{2} \sum_{i=1}^{n} M_{i j}^{2}\left\|e_{i j}(t)\right\|^{2}+\sum_{i=1}^{n} \frac{1}{2} \bar{\varepsilon}_{i j}^{2}\right\}
$$

Substitute (39), (41), controller gain (45), (46) into (53); then, it yields

$$
\dot{V}(t) \leq \sum_{j=1}^{m}\left\{-\frac{1}{2} \sum_{i=1}^{n} \gamma_{i j} \lambda_{\max }(Q)\left\|e_{i j}(t)\right\|^{2}-\frac{1}{2} \sum_{i=1}^{n} \gamma_{i j} \int_{t-\tau_{\max }}^{t} \beta_{i j}\left(x_{i j}(s)\right) d s-\frac{1}{2} \sum_{i=1}^{n} \frac{\kappa_{i j}}{\Gamma_{i j}^{-1}}\left(\widetilde{\omega}_{i j}^{T} \Gamma_{i j}^{-1} \widetilde{\omega}_{i j}\right)+\eta_{j 2}\right\} .
$$



yields

From (21), given the integral inequality in (48), $\dot{V}(t)$

$$
\dot{V}(t) \leq \sum_{j=1}^{m} \sum_{i=1}^{n}\left[-\frac{1}{2} \gamma_{i j} \lambda_{\max }(Q)\left\|e_{i j}(t)\right\|^{2}-\frac{\gamma_{i j}}{2} \int_{t-\tau_{i}(t)}^{t} \beta_{i j}\left(x_{i j}(s)\right) d s-\frac{1}{2} \frac{\kappa_{i j}}{\Gamma_{i j}^{-1}}\left(\widetilde{\omega}_{i j}^{T} \Gamma_{i j}^{-1} \widetilde{\omega}_{i j}\right)+\eta_{j 2}\right] \leq-\eta_{1} V(t)+\eta_{2} .
$$

For $e_{i j} \in \Omega_{\delta_{i}}^{c}$, integrate the above $\dot{V}(t)$ over $[0, t]$; then, it From (21), (37), and (56), the inequality obtains can obtain

$$
0<V(t) \leq \frac{\eta_{2}}{\eta_{1}}+\left(V(0)-\frac{\eta_{2}}{\eta_{1}}\right) e^{-\eta_{1} t}
$$

$$
\sum_{j=1}^{m} \sum_{i=1}^{n} \frac{\lambda_{\min }(Q)}{2}\left\|e_{i j}(t)\right\|^{2} \leq V(t) \leq \frac{\eta_{2}}{\eta_{1}}+\left(V(0)-\frac{\eta_{2}}{\eta_{1}}\right) e^{-\eta_{1} t} \leq \frac{\eta_{2}}{\eta_{1}}+V(0) e^{-\eta_{1} t} \sum_{j=1}^{m} \sum_{i=1}^{n}\left\|e_{i j}(t)\right\|^{2} \leq \frac{2 \eta_{2}}{\lambda_{\min }(D) \eta_{1}}+\frac{2}{\lambda_{\min }(D)} V(0) e^{-\eta_{1} t}
$$

Given $\delta_{i}>\sqrt{2 \eta_{2} / \lambda_{\min }(Q) \eta_{1}}$, there exists $T>0$, for $i=1, \ldots, n$, and all $t>T$ :

$$
\left\|e_{i j}(t)\right\|<\delta_{i}
$$

where $\delta_{i}$ is a small positive constant which determines tracking performance. The inequality can be obtained bounded by the Lyapunov Theory and selecting the appropriate parameters in the closed-loop MASs.

The proof is completed.

Remark 8. For the uncertain nonlinear MASs (1) and (3), Assumptions 1-3, the actual controller is designed in (39) and the adaptive law (41) guarantees that all the signals in the closed-loop systems are SUUB. The parameters selection guidelines for controller design with state time-varying constraints and delays in MASs are demonstrated:

(i) Select appropriately designed parameters such that $k_{i j}^{0}, \omega_{i j}^{T}, \beta_{i j}, \delta_{i}, \Gamma_{i j}, \kappa_{i j}, \gamma_{i j}, \bar{\varepsilon}_{i j}$. Meanwhile, a positive matrix $Q$ is needed to satisfy $Q=P \widehat{\Lambda}^{-1} P^{T}$ and $P \Lambda P^{T}=\widetilde{L} \otimes I_{m}$.

(ii) Decrease the control gain $\Gamma_{i j}, \omega_{i j}^{2}$, and $\bar{\varepsilon}_{i j}$. Increasing the adaptive gain $\gamma_{i j}$ and $k_{i j}^{0}$ would result in better consensus tracking performance. Other parameters are selected according to experience. Therefore, the parameter design of the controller should be adjusted according to the system performance and the tradeoff between tracking performance and constraints between agents such as collision avoidance.

\section{Simulation Example}

The following example is given to illustrate the effectiveness of the proposed adaptive fuzzy tracking control scheme for distributed uncertainties nonlinear MASs containing one leader with three followers. Assume that $i^{\text {th }}$ agent with timevarying delays is as follows:

$$
\left\{\begin{array}{l}
\dot{x}_{i 1}=x_{i 2}(t) \sin \left(D_{i 1} x_{i 1}(t)\right)+\rho_{i 1}\left(x_{i 1}\left(t-\tau_{i}(t)\right)\right)+u_{i}(t), \\
\dot{x}_{i 2}=x_{i 1}(t) \cos \left(D_{i 2} x_{i 2}(t)\right)+\rho_{i 2}\left(x_{i 2}\left(t-\tau_{i}(t)\right)\right)+u_{i}(t) .
\end{array}\right.
$$

The dynamic of the leader can be described as

$$
\dot{x}_{l}(t)=\left[\begin{array}{c}
0.5 \sin (0.8 t) \\
0.1+0.5 \sin (t)
\end{array}\right],
$$

where $u_{i}$ is the input of the cooperative tracking control, $x_{l}(t)$ is the state of the leader, the designed trajectories are $g_{l 1}=0.5 \sin (0.8 t)$, and $g_{l 2}=0.1+0.5 \sin (t)$. The state variables $x_{i 1}(t)$ and $x_{i 2}(t)$ are bounded by time-varying state constraints as follows: $-k_{c_{1}}(t)<x_{i 1}(t)<k_{c_{1}}(t)$, and $k_{c_{1}}(t)$ $=1+0.3 \sin (2 t) ; \quad-k_{c_{2}}(t)<x_{i 2}(t)<k_{c_{2}}(t)$, and $k_{c_{2}}(t)=$ $1.8+0.4 \sin (0.5 t) ; C_{i 1}$ and $C_{i 2}$ are defined in Table 1; and $D_{i 1}$ and $D_{i 2}$ are defined in Table $2, i=1,2,3$. The timevarying delays are $\tau_{1}(t)=1.25-0.5 \sin (0.5 t), \tau_{2}(t)=1.3$ $-0.6 \sin (0.5 t) \sin (t), \tau_{3}(t)=1.2-0.5 \cos (0.5 t), \tau_{\max }=2$.

The information interaction between agents is shown in Figure 2.

The topology illustrates the information interaction between agents in Figure 2 and $B=\operatorname{diag}\{1,1,0\}$. The Laplacian matrix $L$ is given as $L=\left(\begin{array}{ccc}0.7 & -0.7 & 0.0 \\ -0.7 & 1.5 & -0.8 \\ 0.0 & -0.8 & 0.8\end{array}\right)$.

The initial of three agents are $x_{1}(0)=(0.05,0.108)^{T}$, $x_{2}(0)=(-0.13,0.1)^{T}$, and $x_{3}(0)=(0.038,-0.135)^{T}$. The designed parameters are chosen as $\Gamma_{i 1}=\Gamma_{i 2}=1, \kappa_{11}=0.7$, $\kappa_{21}=0.4, \quad \kappa_{31}=0.4, \quad \kappa_{12}=0.6, \quad \kappa_{22}=0.6, \quad \kappa_{32}=0.6$, $k_{0_{1}}=k_{0_{2}}=270, \delta_{i}=10^{-7}$, and $\gamma_{i}=0.9, i=1,2,3$.

Apparently, Assumption 2 can be satisfied by choosing $q_{i}\left(x_{i}(t)\right)=\sqrt{\left(C_{i 1} x_{i 1}(t)\right)^{2}+\left(C_{i 2} x_{i 2}(t)\right)^{2}}$. The adaptive FLSs 
TABle 1: Values of $C_{i 1}$ and $C_{i 2}, i=1,2,3$.

\begin{tabular}{lccc}
\hline$i$ & 1 & 2 & 3 \\
\hline$C_{i 1}$ & 1 & 3 & -2 \\
$C_{i 2}$ & 1.5 & 2.7 & 0.8 \\
\hline
\end{tabular}

TABle 2: Values of $D_{i 1}$ and $D_{i 2}, i=1,2,3$.

\begin{tabular}{lccc}
\hline$i$ & 1 & 2 & 3 \\
\hline$D_{i 1}$ & 0.6 & -3 & 6 \\
$D_{i 2}$ & 0.7 & 0.5 & -5 \\
\hline
\end{tabular}

controller $u_{i}$ is given by (39), and the fuzzy rule numbers are $l_{1}=l_{2}=10$. Choose the fuzzy membership functions as follows:

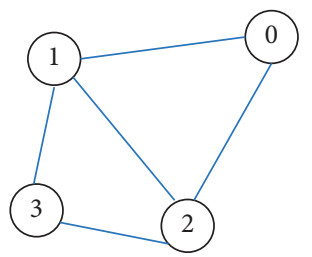

FIGURE 2: The communication topology for one leader and three followers.

$$
\begin{aligned}
& \mu_{F_{i 1}^{l_{1}}}\left(X_{i 1}\right)=\exp \left[-\frac{\left(x_{i 1}-0.5 l_{1}\right)^{2}}{8}-\frac{\left(x_{i 2}-0.5 l_{1}\right)^{2}}{8}-\frac{\left(k_{c_{1}}-0.5 l_{1}\right)^{2}}{8}-\frac{\left(e_{i 1}-0.5 l_{1}\right)^{2}}{8}\right] \\
& \mu_{F_{i 2}^{l_{2}}}\left(X_{i 2}\right)=\exp \left[-\frac{\left(x_{i 1}-0.5 l_{2}\right)^{2}}{8}-\frac{\left(x_{i 2}-0.5 l_{2}\right)^{2}}{8}-\frac{\left(k_{c_{2}}-0.5 l_{2}\right)^{2}}{8}-\frac{\left(e_{i 2}-0.5 l_{2}\right)^{2}}{8}\right] \\
& \Psi_{i 1}^{l_{1}}\left(X_{i 1}\right)=\frac{\mu_{F_{i 1}^{l_{1}}}\left(X_{i 1}\right)}{\sum_{l_{1}=1}^{10} \mu_{F_{i 1}^{l_{1}}}\left(X_{i 1}\right)}, \\
& \Psi_{i 2}^{l_{2}}\left(X_{i 2}\right)=\frac{\mu_{F_{i 1}^{12}}\left(X_{i 2}\right)}{\sum_{l_{2}}^{10} \mu_{F_{i 1}^{l_{2}}}\left(X_{i 2}\right)} .
\end{aligned}
$$

The simulation results are shown in Figures 3-7, and $i=1,2,3$. The trajectories of the desired signal $g_{l 1}$ and full state $x_{i 1}(t)$ with time-varying symmetrical barrier functions $-k_{c_{1}}(t)$ and $k_{c_{1}}(t)$ are shown in Figure 4. The full state $x_{i 2}(t)$ constraints are bounded by time-varying symmetric functions $-k_{c_{2}}(t)$ and $k_{c_{2}}(t)$ and track the desired signal $g_{l 2}$ in Figure 5.

From Figures 3-5, it is easy to indicate that the tracking trajectory always holds within the time-varying constraints $-k_{c^{\prime}}(t)<x_{i j}(t)<k_{c_{j}}(t), j=1,2$, and converges uniformly to the tracking signal. Figure 6 shows the synchronization errors. $e_{i 1}$ and $e_{i 2}$ fluctuate infinitely and approach the origin. Figure 7 shows the norms of the adaptive laws that can show that the effect of control is better. From the simulation, it is obvious that the proposed adaptive fuzzy distributed cooperative tracking control protocol is effective. 


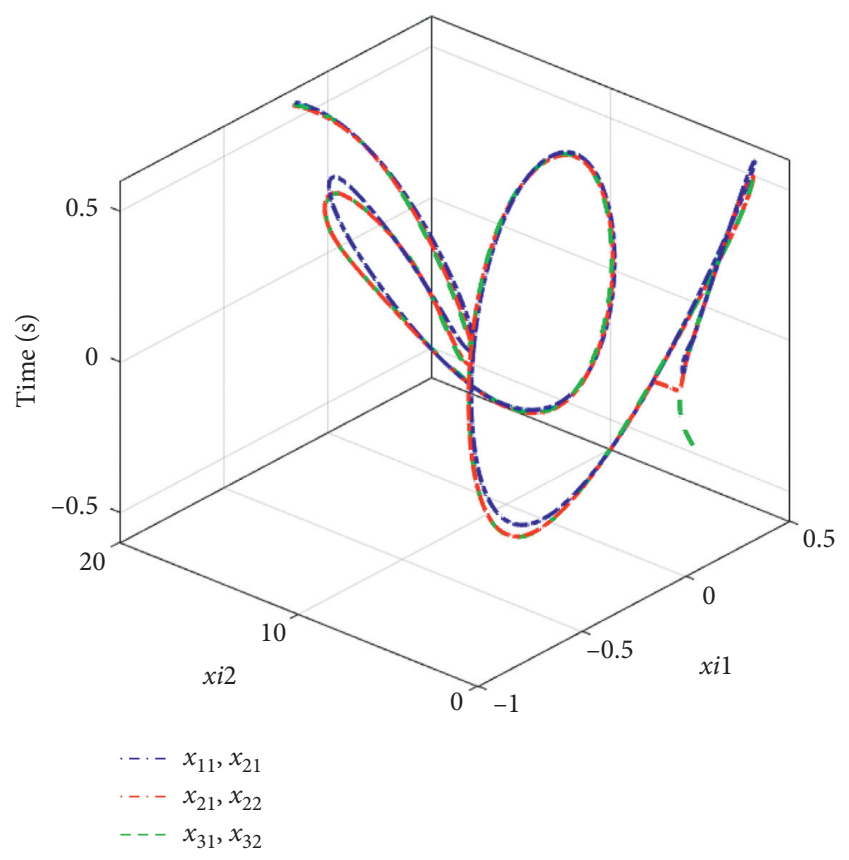

Figure 3: The three agents follow the trajectory of the leader.

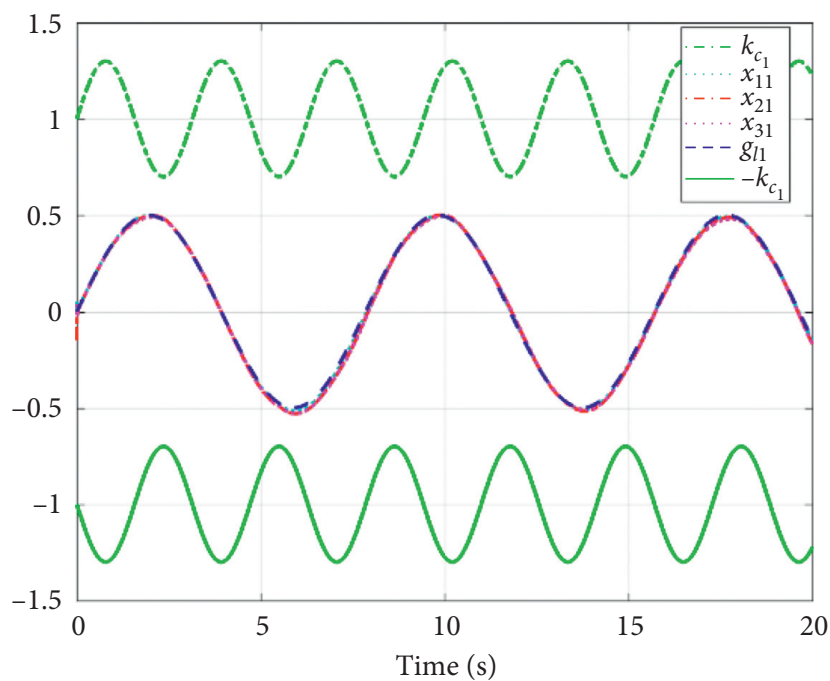

FIGURE 4: The trajectories of state $x_{i 1}$ with the reference signal $g_{l 1}$ in the time-varying constraint boundaries $-k_{c 1}(t)$ and $k_{c 1}(t)$.

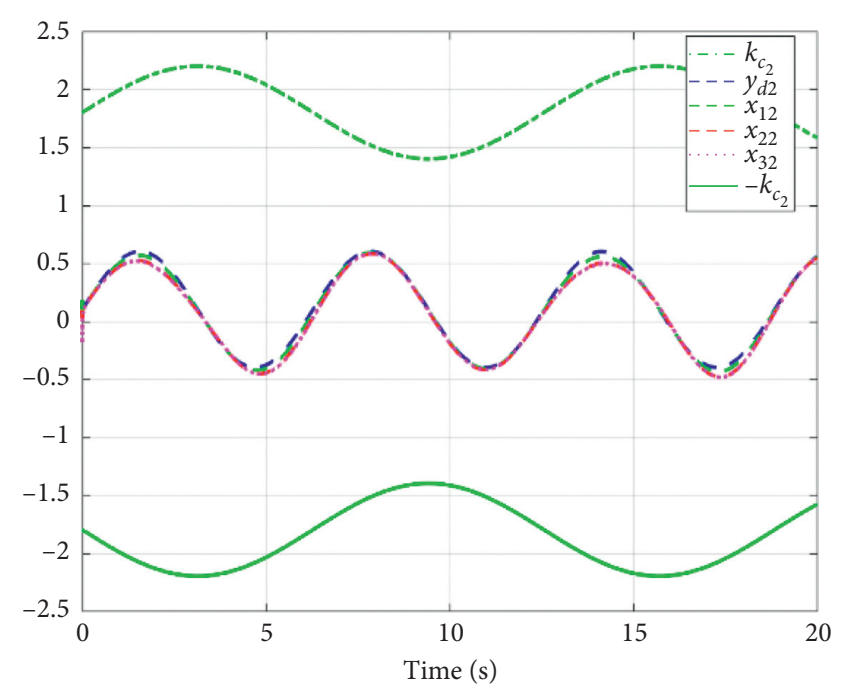

FIGURE 5: The trajectories of state $x_{i 2}$ with the reference signal $g_{l 2}$ in the time-varying constraint boundaries $-k_{c_{2}}(t)$ and $k_{c_{2}}(t)$.
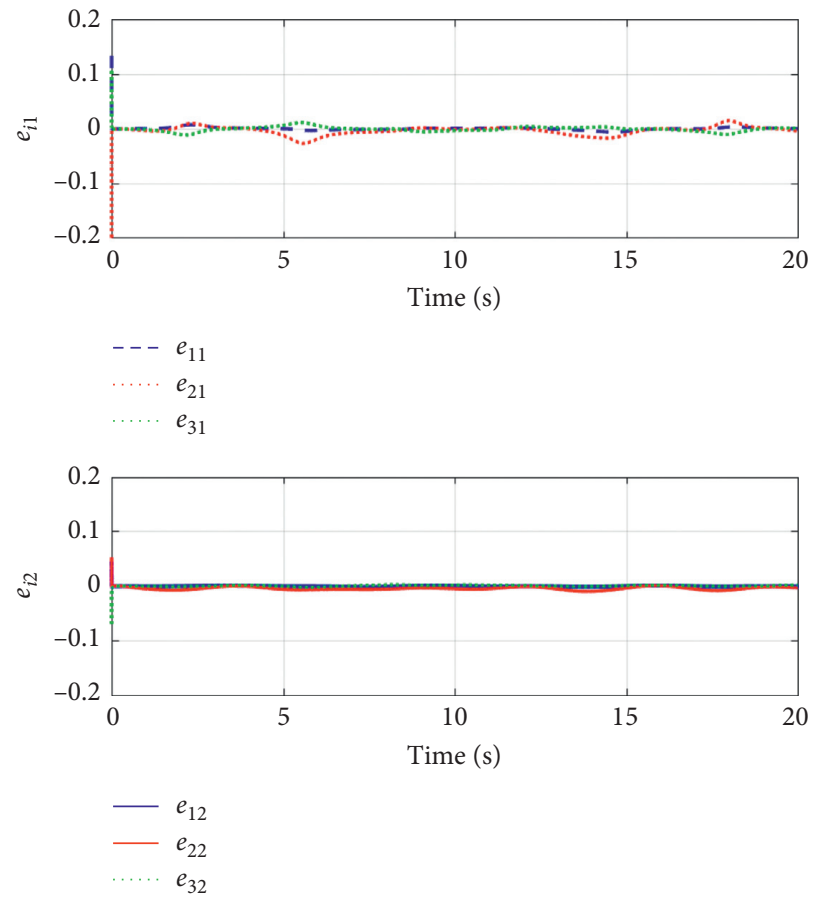

FIgURE 6: The trajectories of errors $e_{i 1}$ and $e_{i 2}$ with time-varying delays and constraints. 

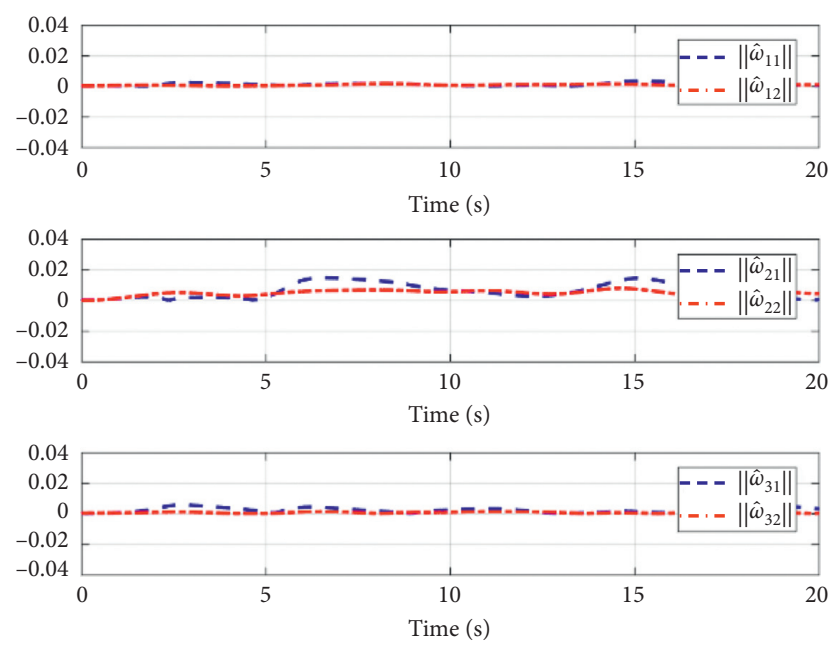

FIgURE 7: The boundedness of the adaptive laws.

\section{Conclusion}

An adaptive fuzzy cooperative tracking control has been presented for a class of uncertain nonlinear MASs with state time-varying delays and time-varying constraints. LKFs have been used to compensate for unknown time-varying delays and overcome the difficulty of employing the variable separation technique in the nonfeedback systems. To guarantee that time-varying delay states would not violate the time-varying bounds by constructing nonlinear coordinate transformations, ulteriorly, additional piecewise constructors can speed up convergence and avoid uncontrollability. The simulation results can be sufficiently demonstrated effective control. This paper further enriches the study of nonlinear state constrained systems. The simulation results provide theoretical support for further practical application.

\section{Data Availability}

The data used to support the findings of this study are included within the article.

\section{Conflicts of Interest}

The authors declare that they have no conflicts of interest.

\section{Acknowledgments}

This work was supported in part by the National Natural Science Foundation of China under Grants 61473139 and 61903168, Provincial Key Research and Development projects under JH2/10200002, and Provincial Scientific Research Fund of Education Department under JJL202015407.

\section{References}

[1] A. Abdessameud and A. Tayebi, "Attitude synchronization of a group of spacecraft without velocity measurements," IEEE
Transactions on Automatic Control, vol. 54, no. 11, pp. 2642-2648, 2009.

[2] H. Zhang, P. Cheng, L. Shi, and J. Chen, "Optimal DoS attack scheduling in wireless networked control system," IEEE Transactions on Control Systems Technology, vol. 24, no. 3, pp. 843-852, 2015.

[3] N. Michael, J. Fink, and V. Kumar, "Cooperative manipulation and transportation with aerial robots," Autonomous Robots, vol. 30, no. 1, pp. 73-86, 2011.

[4] Y. Xu, W. Liu, and J. Gong, "Stable multi-agent-based load shedding algorithm for power systems," IEEE Transactions on Power Systems, vol. 26, no. 4, pp. 2006-2014, 2011.

[5] W. Meng, X. Wang, and S. Liu, "Distributed load sharing of an inverter-based microgrid with reduced communication," IEEE Transactions on Smart Grid, vol. 9, no. 2, pp. 1354-1364, 2016, to be published.

[6] B. Grocholsky, J. Keller, V. Kumar, and G. Pappas, "Cooperative air and ground surveillance," IEEE Robotics and Automation Magazine, vol. 13, no. 3, pp. 16-25, 2006.

[7] I.-S. Jeon, J.-I. Lee, and M.-J. Tahk, "Homing guidance law for cooperative attack of multiple missiles," Journal of Guidance, Control, and Dynamics, vol. 33, no. 1, pp. 275-280, 2010.

[8] F. De Rango, G. Potrino, M. Tropea, A. F. Santamaria, and P. Fazio, "Scalable and ligthway bio-inspired coordination protocol for FANET in precision agriculture applications," Computers and Electrical Engineering, vol. 74, pp. 305-318, 2019.

[9] H. Su, X. Wang, and Z. Lin, "Flocking of multi-agents with a virtual leader," IEEE Transactions on Automatic Control, vol. 54, no. 2, pp. 293-307, 2009.

[10] W. Ren, R. W. Beard, and E. M. Atkins, "Information consensus in multivehicle cooperative control," IEEE Control Systems, vol. 27, no. 2, pp. 71-82, 2007.

[11] Z. Li, X. Liu, W. Ren, and L. Xie, "Distributed tracking control for linear multiagent systems with a leader of bounded unknown input," IEEE Transactions on Automatic Control, vol. 58, no. 2, pp. 518-523, 2012.

[12] G. Shi and Y. Hong, "Global target aggregation and state agreement of nonlinear multi-agent systems with switching topologies," Automatica, vol. 45, no. 5, pp. 1165-1175, 2009.

[13] G. Wen, C. P. Chen, and Y. J. Liu, "Formation control with obstacle avoidance for a class of stochastic multiagent systems," IEEE Transactions on Industrial Electronics, vol. 65, no. 7, pp. 5847-5855, 2017.

[14] R. Olfati-Saber, J. A. Fax, and R. M. Murray, "Consensus and cooperation in networked multi-agent systems," Proceedings of the IEEE, vol. 95, no. 1, pp. 215-233, 2007.

[15] R. Olfati-Saber, "Flocking for multi-agent dynamic systems: algorithms and theory," IEEE Transactions on Automatic Control, vol. 51, no. 3, pp. 401-420, 2006.

[16] J. Gao, J. Liu, B. Rajan et al., "SCADA communication and security issues," Security and Communication Networks, vol. 7, no. 1, pp. 175-194, 2014.

[17] P. Ping Guo, C. L. P. Chen, and M. R. Lyu, "Cluster number selection for a small set of samples using the Bayesian YingYang model," IEEE Transactions on Neural Networks, vol. 13, no. 3, pp. 757-763, 2002.

[18] W. Cao, Y. Zhou, C. L. P. Chen, and L. Xia, "Medical image encryption using edge maps," Signal Processing, vol. 132, pp. 96-109, 2017.

[19] S. Sui, C. L. P. Chen, and S. Tong, "Neural-Network-based adaptive DSC design for switched fractional-order nonlinear systems," IEEE Transactions on Neural Networks and Learning Systems, pp. 1-10, 2020. 
[20] L. Liu, X. Li, Y.-J. Liu, and S. Tong, "Neural network based adaptive event trigger control for a class of electromagnetic suspension systems," Control Engineering Practice, vol. 106, Article ID 104675, 2021.

[21] S. Sui, C. L. P. Chen, and S. Tong, "Event-trigger-based finitetime fuzzy adaptive control for stochastic nonlinear system with unmodeled dynamics," IEEE Transactions on Fuzzy Systems, p. 1, 2020.

[22] H. A. Yousef, M. Hamdy, A. Saleem, K. Nashed, M. Mesbah, and M. Shafiq, "Enhanced adaptive control for a benchmark piezoelectric-actuated system via fuzzy approximation," International Journal of Adaptive Control and Signal Processing, vol. 33, no. 9, pp. 1329-1343, 2019.

[23] H. A. Yousef, M. Hamdy, and K. Nashed, "L1 adaptive fuzzy controller for a class of nonlinear systems with unknown backlash-like hysteresis," International Journal of Systems Science, vol. 48, no. 12, pp. 2522-2533, 2017.

[24] Q. Shen, B. Jiang, P. Shi, and J. Zhao, "Cooperative adaptive fuzzy tracking control for networked unknown nonlinear multiagent systems with time-varying actuator faults," IEEE Transactions on Fuzzy Systems, vol. 22, no. 3, pp. 494-504, 2013.

[25] G. X. Wen, C. L. P. Chen, Y. J. Liu, and Z. Liu, "Neuralnetwork-based adaptive leader-following consensus control for second-order non-linear multi-agent systems," IET Control Theory \& Applications, vol. 13, no. 9, pp. 1927-1934, 2015.

[26] H. Zhang and F. L. Lewis, "Adaptive cooperative tracking control of higher-order nonlinear systems with unknown dynamics," Automatica, vol. 48, no. 7, pp. 1432-1439, 2012.

[27] W. Wang and S. Tong, "Adaptive fuzzy containment control of nonlinear strict-feedback systems with full state constraints," IEEE Transactions on Fuzzy Systems, vol. 27, no. 10, pp. 2024-2038, 2019.

[28] S. S. Ge, F. Hong, and T. H. Lee, "Adaptive neural control of nonlinear time-delay systems with unknown virtual control coefficients," IEEE Transactions on Systems, Man and Cybernetics, Part B (Cybernetics), vol. 34, no. 1, pp. 499-516, 2004.

[29] Z. Liu, G. Lai, Y. Zhang, and C. L. P. Chen, “Adaptive fuzzy tracking control of nonlinear time-delay systems with deadzone output mechanism based on a novel smooth model," IEEE Transactions on Fuzzy Systems, vol. 23, no. 6, pp. 1998-2011, 2015.

[30] D. Yang, X. Li, J. Shen, and Z. Zhou, "State-dependent switching control of delayed switched systems with stable and unstable modes," Mathematical Methods in the Applied Sciences, vol. 41, no. 16, pp. 6968-6983, 2018.

[31] Y. Dan, X. D. Li, and J. L. Qiu, "Output tracking control of delayed switched systems via state-dependent switching and dynamic output feedback," Nonlinear Analysis: Hybrid Systems, vol. 32, pp. 294-305, 2019.

[32] C. L. P. Chen, G. X. Wen, Y. J. Liu, and F. Wang, "Adaptive consensus control for a class of nonlinear multiagent timedelay systems using neural networks," IEEE Transactions on Neural Networks and Learning Systems, vol. 25, pp. 1217-1226, 2017.

[33] G. Wen, C. L. P. Chen, Y.-J. Liu, and Z. Liu, "Neural networkbased adaptive leader-following consensus control for a class of nonlinear multiagent state-delay systems," IEEE Transactions on Cybernetics, vol. 47, no. 8, pp. 2151-2160, 2017.

[34] K. P. Tee, S. S. Ge, and E. H. Tay, "Barrier Lyapunov functions for the control of output-constrained nonlinear systems," Automatica, vol. 45, no. 4, pp. 918-927, 2009.
[35] Z. Zhi Liu, G. Y. Guanyu Lai, Y. Yun Zhang, and C. L. P. Chen, "Adaptive neural output feedback control of output-constrained nonlinear systems with unknown output nonlinearity," IEEE Transactions on Neural Networks and Learning Systems, vol. 26, no. 8, pp. 1789-1802, 2015.

[36] S. Sui, C. L. P. Chen, and S. Tong, "A novel adaptive NN prescribed performance control for stochastic nonlinear systems," IEEE Transactions on Neural Networks and Learning Systems, pp. 1-10, 2020.

[37] Y.-J. Liu, Q. Zeng, S. Tong, C. L. P. Chen, and L. Liu, "Adaptive neural network control for active suspension systems with time-varying vertical displacement and speed constraints," IEEE Transactions on Industrial Electronics, vol. 66, no. 12, pp. 9458-9466, 2019.

[38] J. Lan, Y.-J. Liu, L. Liu, and S. Tong, "Adaptive output feedback tracking control for a class of nonlinear time-varying state constrained systems with fuzzy dead-zone input," IEEE Transactions on Fuzzy Systems, p. 1, 2020.

[39] Y. J. Liu, S. C. Tong, C. L. P. Chen, and D. J. Li, “Adaptive NN control using integral barrier Lyapunov functionals for uncertain nonlinear block-triangular constraint systems," IEEE Transactions on Cybernetics, vol. 47, no. 11, pp. 3747-3757, 2016.

[40] L. Liu, Y. J. Liu, A. Chen, S. C. Tong, and C. L. P. Chen, "Integral barrier Lyapunov function-based adaptive control for switched nonlinear systems," Science China Information Sciences, vol. 63, no. 3, pp. 1-14, 2020.

[41] L. Liu, T. Gao, Y.-J. Liu, S. Tong, C. L. P. Chen, and L. Ma, "Time-varying IBLFs-based adaptive control of uncertain nonlinear systems with full state constraints," Automatica, vol. 129, Article ID 109595, 2021.

[42] Y. Zhang, H. Liang, H. Ma, Q. Zhou, and Z. Yu, "Distributed adaptive consensus tracking control for nonlinear multi-agent systems with state constraints," Applied Mathematics and Computation, vol. 326, pp. 16-32, 2018.

[43] K. Zhao and Y. Song, "Removing the feasibility conditions imposed on tracking control designs for state-constrained strict-feedback systems," IEEE Transactions on Automatic Control, vol. 64, no. 3, pp. 1265-1272, 2019.

[44] W. Meng, Q. Yang, J. Si, and Y. Sun, "Consensus control of nonlinear multiagent systems with time-varying state constraints," IEEE Transactions on Cybernetics, vol. 47, no. 8, pp. 2110-2120, 2016.

[45] D.-P. Li, D.-J. Li, Y.-J. Liu, S. Tong, and C. L. P. Chen, "Approximation-based adaptive neural tracking control of nonlinear MIMO unknown time-varying delay systems with full state constraints," IEEE Transactions on Cybernetics, vol. 47, no. 10, pp. 3100-3109, 2017.

[46] D. Li, C. L. P. Chen, Y.-J. Liu, and S. Tong, "Neural network controller design for a class of nonlinear delayed systems with time-varying full-state constraints," IEEE Transactions on Neural Networks and Learning Systems, vol. 30, no. 9, pp. 2625-2636, 2019. 